\begin{tabular}{|c|c|}
\hline Balkanologie & Balkanologie \\
\hline & Vol. $15 n^{\circ} \mathbf{2} \mid \mathbf{2 0 2 0}$ \\
\hline
\end{tabular}

\title{
Dilemmes de genre postsocialistes : la Marche des lesbiennes de Belgrade de 2015 et ses antécédents
}

Post-Socialist Gender Dilemmas: 2015 Belgrade Lesbian March and its Antecedents

\section{Bojan Bilić}

Traducteur : Amaël Cattaruzza, Cécile Jouhanneau, Sarah Sajn et Detelina Tocheva

\section{(2) OpenEdition}

\section{Journals}

Édition électronique

URL : http://journals.openedition.org/balkanologie/2605

DOI : 10.4000/balkanologie.2605

ISSN : 1965-0582

Éditeur

Association française d'études sur les Balkans (Afebalk)

Référence électronique

Bojan Bilić, «Dilemmes de genre postsocialistes : la Marche des lesbiennes de Belgrade de 2015 et ses antécédents », Balkanologie [En ligne], Vol. 15 n² | 2020, mis en ligne le 01 décembre 2020, consulté le 26 avril 2021. URL : http://journals.openedition.org/balkanologie/2605 ; DOI : https:// doi.org/10.4000/balkanologie.2605

Ce document a été généré automatiquement le 26 avril 2021.

(c) Tous droits réservés 


\title{
Dilemmes de genre postsocialistes : la Marche des lesbiennes de Belgrade de 2015 et ses antécédents
}

\author{
Post-Socialist Gender Dilemmas: 2015 Belgrade Lesbian March and its
}

Antecedents

Bojan Bilić

Traduction : Amaël Cattaruzza, Cécile Jouhanneau, Sarah Sajn et Detelina Tocheva

\section{NOTE DE L'ÉDITEUR}

Cet article est une version adaptée et traduite d'un texte paru dans l'ouvrage : BILIĆ Bojan, Trauma, Violence, and Lesbian Agency in Croatia and Serbia: Building Better Times, Londres, Palgrave Macmillan, 2020, p. 125-159, réalisée avec l'autorisation de Springer Nature/Palgrave Macmillan.

\section{NOTE DE L'AUTEUR}

L'auteur tient à remercier les traductrices et le traducteur ainsi que Mladen Ostojić et Inès Kerboua pour leurs commentaires sur le texte.

1 Après près de deux décennies très conflictuelles et violentes, 2015 s'est avérée être une annus mirabilis dans la mobilisation des militants serbes et post-yougoslaves non hétérosexuels et transsexuels. Cette année-là, non seulement le défilé de la Fierté (Pride Parade) de Belgrade s'est relativement bien déroulé, mais les rues de la capitale serbe (précédemment également yougoslave) ont pour la première fois accueilli la Marche des lesbiennes et de la Fierté trans. D'une part, cette diversité d'évènements témoigne de la vitalité des courants militants LGBT qui ont survécu à de longues périodes d'homophobie institutionnalisée et socialement répandue. D'autre part, elle souligne 
l'existence de tensions, de divisions et de défis internes auxquels les militants doivent faire face dans leur lutte pour l'émancipation LGBT.

2 La Marche des lesbiennes de Belgrade de 2015 a représenté le point culminant des courants séparatistes qui se sont développés de manière timide mais soutenue dans l'espace post-yougoslave au cours des dernières décennies ${ }^{1}$. Dans cet article, je mappuie sur diverses sources empiriques - incluant des vidéos YouTube, des déclarations publiques, des articles de journaux, des interviews en ligne et mes propres entretiens avec des militants, ainsi que des discussions au sein du groupe Google « razotkvirivanje ${ }^{2} »-$ pour reconstituer la Marche de 2015. Mon objectif est d'illustrer comment le séparatisme lesbien - une forme radicale de militantisme lesbien dans laquelle les activités sont menées " par des femmes pour des femmes » et excluent donc les hommes - est entré dans l'espace yougoslave plus explicitement que jamais auparavant, provoquant un ensemble de controverses similaires à celles qui l'accompagnent dans d'autres parties du monde. Pour ce faire, je replace dans son contexte ce rassemblement public inhabituel, le premier du genre dans l'espace postyougoslave, à la fois dans l'histoire de l'activisme féministe/lesbien yougoslave et dans l'histoire plus large de l'activisme lesbien (et GBT) anglo-américain. Ce dernier exerce une influence mondiale sur les initiatives militantes car les stratégies de protestation sont globalement initiées (principalement) depuis les États-Unis et incorporées - par un processus de traduction ${ }^{3}$ troublé - dans la logique des structures politiques nationales (semi-périphériques).

3 J'affirme que la Marche lesbienne de 2015 a été conçue comme un exemple de ce que Della Porta appelle le cracking : elle était censée représenter une rupture soudaine de la routine dans le but de remettre en question l'ordre établi et de produire un effet historique ${ }^{4}$. À cet égard, la Marche a été un acte de visibilité lesbienne, organisé en réponse à la marginalisation générale des activistes lesbiennes ainsi qu'à la lesbophobie et à la misogynie généralisées dans le contexte social et politique post-yougoslave. En tant que manifestation de rupture (cracking), la Marche a été un événement extraordinaire qui a renforcé la présence des lesbiennes dans la vie publique, soulignant la force des liens entre militantes lesbiennes au niveau régional et européen. La Marche a constitué et dévoilé un contre-public subalterne ${ }^{5}$ qui a non seulement dilaté la sphère privée pour exposer ce qui jusqu'alors n'était pas suffisamment visible, mais a servi de plateforme à partir de laquelle de nouvelles identités ont pu être expérimentées, formées et mises en œuvre. En élargissant le champ de ce qui peut être dit par le biais de slogans lesbiens explicites criés dans la rue, les militantes ont lutté pour convaincre les autres - et se convaincre elles-mêmes - que «ce qui n'était pas public dans le passé, au sens où il s'agissait d'une préoccupation commune, devrait maintenant le devenir $»^{6}$.

4 Cependant, les revendications du « droit à la ville $»^{7}$ des lesbiennes sont loin de l'idéal unifié présent dans l'œuvre de Fraser, car les contre-publics lesbiens sont multiples et divisés ${ }^{8}$. La Marche a révélé des clivages profonds parmi les groupes d'activistes et entre les activistes, principalement en ce qui concerne la manière dont les organisatrices ont inclus ou exclu les femmes (lesbiennes), les hommes et les femmes transgenres. La décision des organisatrices de ne pas autoriser les participants masculins (dans une manifestation "protégée » par des policiers) a perturbé la nature publique de l'espace public, en sapant l'effort visant à accroître l'attention du public à la 
cause lesbienne et en résolvant de façon radicale la question du rôle que les hommes devraient jouer dans les luttes féministes.

5 Pour examiner comment cette stratégie problématique est devenue possible, j'ai rapproché la Marche d'autres initiatives féministes et lesbiennes, en particulier le rassemblement international féministe de Belgrade de 1978 intitulé «Camarade femme » (Drug-ca žena). Lors de cette réunion, la question de la participation masculine a été un point de discorde, divisant les participantes à la conférence selon une ligne capitalisme-socialisme qui s'avérerait pertinente pour les décennies à venir. À la fin des années 1970, ce clivage reflétait des différences idéologiques notables, conditionnées par les différentes réalités sociales des participantes - à savoir, vivre dans un État socialiste ou capitaliste - qui, du fait du démantèlement rapide des acquis socialistes et de l'affaiblissement global de l'État-providence vers la fin du $\mathrm{xx}^{\mathrm{e}}$ siècle, sont devenues de plus en plus similaires. De tels processus politiques ont, en retour, ouvert la voie à une application parfois peu critique des modèles d'activisme anglosaxons, ayant pour effet de maintenir la région dans la « deuxième vague féministe » et de l'empêcher de tirer parti des luttes émancipatrices locales. La réification de la binarité de genre, base structurelle de la discrimination par le genre, a dévoilé le caractère contrerévolutionnaire de la "transition" post-yougoslave, démontrant le triomphe de la politique identitaire propre au modèle néolibéral.

Dans la première partie de l'article, je présente le contexte idéologique de la Marche en discutant de certaines des dimensions les plus importantes du séparatisme lesbien tel qu'il s'est développé aux États-Unis. Je reviens ensuite sur la conférence «Camarade femme » qui a marqué l'entrée de la deuxième vague féministe en Europe de l'Est, en montrant comment elle a agi comme un «point de bifurcation» entre les sexes qui bien qu'implicitement - a introduit l'idée du séparatisme lesbien et des initiatives exclusivement féminines dans l'espace yougoslave. J'aborde également d'autres formes d'organisation féministe et lesbienne basées à Belgrade (par exemple, la création du groupe militant lesbien Labris) pour affirmer que, depuis la conférence de 1978, le séparatisme lesbien a été un fil conducteur idéologique qui a guidé les efforts des militants lesbiens qui ont culminé avec la Marche de 2015. S'ensuit une analyse plus empirique qui présente la logique des organisatrices de la Marche ainsi que les réactions de ceux/celles qui pensaient que le combat pour accroître la visibilité des lesbiennes aurait pu bénéficier davantage de stratégies militantes alternatives. Ici, j'analyse surtout le débat que l'annonce de la Marche et son déroulement ultérieur ont suscité au sein du groupe Google razotkvirivanje. Dans la dernière partie de l'article, j'examine les implications des stratégies mises en place par les activistes séparatistes pour la libération non hétérosexuelle dans le contexte postsocialiste.

\section{Le séparatisme lesbien, un paria rebelle}

7 Selon Julia R. Enszer, bien qu'il soit rarement pris au sérieux, y compris dans les discours féministes contemporains, « les racines intellectuelles du séparatisme lesbien sont riches, profondes et complexes ${ }^{9} »$. Le séparatisme lesbien est apparu sous ses formes les plus explicites au cours de la décennie 1970, dans le sillage des mobilisations étudiantes internationales des années 1968 qui s'inscrivaient dans des contextes nationaux singuliers et qui ont inclus, dans une plus ou moins grande mesure, une « révolution sexuelle» ayant bouleversé les normes sexuelles traditionnelles. Dans sa 
tentative d'articuler la théorie politique et les pratiques féministes concrètes, le séparatisme lesbien a représenté un processus consistant à privilégier les femmes et les lesbiennes, une façon d'être et d'interagir dans le monde qui «promettait à ses praticiennes une vie de rébellion radicale et d'empowerment féministe $»^{10}$.

Une des premières définitions du séparatisme lesbien provient du manifeste «La femme identifiée comme femme" publié en 1970 par le groupe militant

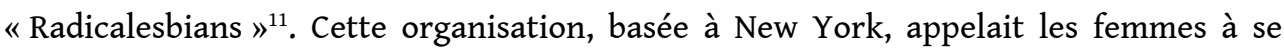
concentrer sur «la primauté des femmes s'engageant avec des femmes, des femmes créant une nouvelle conscience de l'autre et avec l'autre, qui est au cœur de la libération des femmes, et à la base de la révolution culturelle». C'est ce que fit Lois Anne Addison en 1971 lorsqu'elle lança avec sa compagne le journal clandestin Spectre dans lequel elles se présentaient comme des «lesbiennes révolutionnaires » en lutte pour " une société communiste qui ne soit pas fondée sur l'exploitation ». Dans un court article publié dans le deuxième numéro du journal et intitulé «Comment cesser de mourir étouffées ", elles prônaient le séparatisme défini comme le fait de "travailler directement seulement avec des femmes ».

9 La ferveur séparatiste atteignit son apogée avec la création d'un groupe lesbien radical baptisé le «Collectif des furies ». Les douze femmes qui le composaient commencèrent à publier en 1972 le mensuel The Furies et déclaraient dans leur manifeste :

Nous sommes en colère car nous sommes opprimées par la suprématie masculine. Toute notre vie nous avons été baisées par un système qui est fondé sur la domination des femmes par les hommes et qui définit le masculin comme bon et le féminin seulement comme aussi bon que l'est l'homme avec qui vous vous trouvez. C'est un système dans lequel l'hétérosexualité est strictement imposée et le lesbianisme strictement supprimé. C'est un système qui nous a en outre divisées selon la classe, la race et la nationalité.

10 Dans ce texte, elles identifiaient les enjeux les plus saillants du séparatisme : le fait que la lesbianité soit un choix politique nécessaire - un impératif politique - pour les féministes, une identité idoine pour toutes membres du mouvement des femmes dans une société fondée sur le suprématisme masculin et dans laquelle l'hétérosexualité était érigée en institution politique. Le manifeste soulignait aussi l'échec du "mouvement des femmes hétérosexuelles » et de la "gauche masculine » à prendre en charge les préoccupations lesbiennes et insistait sur la nécessité pour les lesbiennes de construire une " politique commune» du "lesbianisme comme question politique ${ }^{12} »$. En ce sens, pour les militantes séparatistes, le séparatisme n'était pas considéré comme un refus mais comme le lieu d'une expérimentation potentiellement productive politiquement au sein de communautés exclusivement féminines. Adrienne Rich, en tant que corédactrice en cheffe d'un autre journal séparatiste intitulé Sinister Wisdom, écrivait que :

Un acte de séparation, le fait d'être séparées, peut aussi être un acte de connexion. Un espace entièrement féminin n'est pas définissable seulement comme un lieu d'où les hommes sont exclus. Il peut aussi être - bien que ce ne soit pas toujours le cas - un lieu où des femmes qui ne se sont pas entendues auparavant témoignent (pour reprendre l'expression de Sarah Hoagland) en présence les unes des autres et se décrivent aux autres, forgeant ainsi de nouveaux liens de confiance et ouvrant de nouvelles possibilités de mouvement. ${ }^{13}$

11 L'un des événements les plus connus de mise en pratique du raisonnement séparatiste lesbien était le Michigan Womyn’s Music Festival (« Michfest»), créé en 1976 et actif pendant près de 40 ans, jusqu'à 2015. Le but du festival était d'offrir aux femmes un lieu 
où elles pouvaient se retrouver. Tout le travail nécessaire pour le produire était réalisé exclusivement par des femmes. Le Michfest a changé la vie de nombre de ses participantes, mais il n'est pas allé sans son lot de controverses. La question la plus polémique, à savoir la participation des femmes trans (qui n'étaient pas admises dans un festival ouvert seulement aux "femmes nées femmes»14, a accompagné le séparatisme lesbien dès ses débuts et, bien au-delà des États-Unis, dans le monde entier.

Bien que de nombreux événements séparatistes ultérieurs aient été ouverts aux femmes trans (y compris la Marche de Belgrade, du moins en théorie), le séparatisme lesbien suscite régulièrement des controverses, en particulier autour de la définition de la «femme ». En ce sens, la politique séparatiste a rencontré de sérieuses critiques au sein même des cercles féministes, et ce dès les années 1970. La critique provenait en particulier des féministes noires (ce qui ne signifie pas qu'aucune femme noire ne se soit reconnue dans le séparatisme), en particulier du "Combahee River Collective, » une organisation lesbienne féministe noire active à Boston entre 1974 et 1980. Ses membres considéraient le séparatisme lesbien comme une idéologie raciste et affirmaient dans leur Manifeste féministe noir que les hommes ne devaient pas être exclus des luttes militantes progressistes.

Bien que nous soyons féministes et lesbiennes, nous nous sentons solidaires des hommes noirs progressistes et nous ne prônons pas la fractionnalisation que réclament les femmes blanches qui sont séparatistes. Notre situation comme personnes noires rend nécessaire la solidarité autour du fait racial, une solidarité bien sûr inutile pour les femmes blanches envers les hommes blancs, à moins qu'il ne s'agisse d'une solidarité négative entre oppresseurs de race. Nous luttons au coude à coude avec les hommes noirs contre le racisme, tout en luttant aussi avec eux par rapport au sexisme. (...) nous rejetons la position du séparatisme lesbien, qui n'est ni une analyse, ni une stratégie politique viable pour nous. Il laisse dehors beaucoup trop de personnes, en particulier les hommes, les femmes et les enfants noir $\bullet \bullet$ e. Nous avons beaucoup de critiques et d'aversion envers ce que les hommes ont été socialisés à être dans cette société: envers ce qu'ils soutiennent, leur manière d'agir et d'opprimer. Mais nous ne nous égarons pas à croire que c'est leur masculinité en soi, c'est-à-dire leur masculinité biologique, qui fait d'eux ce qu'ils sont. En tant que femmes noires, nous estimons que n'importe quel déterminisme biologique constitue une base politique dangereuse et réactionnaire. Nous devons aussi nous demander si le séparatisme lesbien peut constituer une analyse et une stratégie adaptée et progressiste, même pour celles qui la pratiquent, dans la mesure où elle dénie complètement toutes les sources de l'oppression des femmes autres que sexuelle, niant par là-même les faits de classe et de race..$^{15}$

13 Tandis que les premières séparatistes lesbiennes féministes états-uniennes blanches se déclaraient communistes et imaginaient des communautés exclusivement féminines, les membres du Combahee River Collective se définissaient comme des socialistes prônant une révolution socialiste qui soit à la fois féministe et antiraciste. Quelle que soit la distinction entre le communisme et le socialisme, il est important de souligner l'orientation politique de gauche prononcée de l'activisme lesbien féministe radical opérant dans la société capitaliste des années 1970. C'était une époque où la lesbianité était désignée comme "la menace mauve» par les meneuses du mouvement des femmes, ce qui lui conférait « une connotation contre-culturelle si puissante que les femmes étaient attirées vers elle par idéologie plus que par désiri" ${ }^{16}$.

14 En outre, le séparatisme lesbien a émergé en réponse non seulement au manque de visibilité des lesbiennes au sein du mouvement des femmes, mais aussi à l'échec du 
terme "gay" à englober les hommes homosexuels, les lesbiennes, les personnes bisexuelles et plus largement non hétérosexuelles. Au lieu de constituer un dénominateur commun, depuis les années 1970, le terme «gay" désigne principalement les hommes homosexuels ${ }^{17}$. De façon similaire, durant l'épidémie de SIDA dans les années 1980, la forte association entre le mouvement de lutte contre le SIDA et les hommes a une fois de plus éclipsé les communautés lesbiennes. Au début des années 1990, le courant dominant du mouvement de la Gay Pride était en train de devenir principalement masculin, blanc et de classe moyenne, professionnalisé, institutionnalisé, hyper-sexualisé, dépolitisé et commercialisé, ce qui menaçait de renforcer les asymétries de genre au sein des initiatives militantes ${ }^{18}$. Pour contrer cette tendance, quelques groupes lesbiens menés par les «Lesbian Avengers of New York City ", une organisation fondée en 1992, lancèrent une marche lesbienne séparée au sein de la Marche sur Washington pour l'égalité des droits et la libération des lesbiennes, gays et bi de 1993. On rapporte que 20000 femmes participèrent à cet événement qui, s'il ne constituait pas la première manifestation lesbienne publique en Amérique du Nord, a toutefois été la première qui soit parvenue à générer un mouvement ${ }^{19}$. Durant les décennies suivantes, cette manifestation a représenté un modèle pour les militantes lesbiennes et a inspiré des rassemblements similaires aux États-Unis, en Amérique du Nord et en Europe de l'Ouest. Les manifestations lesbiennes étaient censées offrir une alternative à la Marche des fiertés et réussir là où cette dernière avait échoué - à savoir, représenter des espaces inclusifs de contestation du patriarcat, du capitalisme, de la consommation de masse, de la professionnalisation et de l'intense dépolitisation des questions LGBT ${ }^{20}$.

\section{Un tournant : la conférence de 1978 « Camarade femme »}

Les trajectoires du séparatisme lesbien dans l'espace yougoslave mènent également aux années 1970. Du 27 au 29 octobre 1978, Belgrade a accueilli un groupe international d'universitaires et de militants et militantes féministes qui, avec leurs hôtes yougoslaves, pour la plupart des universitaires de Belgrade et Zagreb, ont participé à la conférence "Camarade femme ", sous-titrée "La question des femmes: une nouvelle approche? » (Drug-ca žena. Žensko pitanje: novi pristup?). Ce rassemblement a, comme la Marche des lesbiennes, été considéré comme un événement international hors normes et a renforcé les liens entre les militants de la région. Il a en effet marqué le début du mouvement féministe socialiste yougoslave autonome de l'après Seconde Guerre mondiale ${ }^{21}$ et l'émergence de la « deuxième vague du féminisme » en Europe de l'Est. Il a constitué une occasion sans précédent pour les féministes de régimes politiques et économiques différents, à savoir le socialisme et le capitalisme, d'échanger leurs points de vue sur le statut des femmes (la « question féminine ») dans ces contextes respectifs. Comme s'en souvient un participant :

C'était le premier événement de type conférence avec un agenda féministe, essayant de réunir des gens de Belgrade et de Zagreb et d'ailleurs en Yougoslavie, ainsi que beaucoup de gens de l'étranger. Il y a eu environ 80 personnes pendant ces deux jours. Le noyau du groupe féministe a donc essayé de sortir de ce cercle intellectuel très restreint pour l'élargir un peu plus, amener quelques jeunes étudiants et attirer l'attention des médias. C'était une réunion d'exploration et de discussion autour d'un agenda commun, basé sur un travail en réseau... et c'était 
fascinant de faire venir toutes ces personnes de l'étranger, toutes d'orientations très différentes. C'était dynamique et polémique mais, bien sûr, tout cela a été ralenti par les traductions. ${ }^{22}$ internationaux était principalement due au fait que la législation progressiste de l'État socialiste yougoslave avait déjà anticipé des mesures d'émancipation (dont certaines très importantes) exigées par les mobilisations féministes autonomes en Europe occidentale. Par exemple, l'avortement était autorisé et réglementé en Yougoslavie depuis 1952 (avec l'idée de réduire les avortements illégaux qui, à l'époque, entraînaient une mortalité élevée), alors qu'il n'est devenu légal en Italie qu'en mai 1978 - l'année de la conférence - grâce à l'engagement de longue date des militantes féministes, dont certaines étaient présentes à Belgrade. Il semble que les invités internationaux n'aient pas suffisamment apprécié le fait que le régime socialiste - au moins de manière déclarative et légale - ait placé l'émancipation des femmes en tête de son programme politique. L'article 24 de la Constitution de 1946 de la République populaire fédérative de Yougoslavie introduisait déjà des dispositions légales qui ne sont toujours pas en vigueur dans certaines démocraties actuelles :

Les femmes ont les mêmes droits que les hommes dans tous les domaines de la vie étatique, économique et sociopolitique. Les femmes ont droit au même salaire que celui perçu par les hommes pour le même travail et, en tant que travailleuses ou employées, elles bénéficient d'une protection spéciale. L'État protège tout particulièrement les intérêts des mères et des enfants par la création de maternités, de foyers pour enfants et de crèches et par le droit des mères à un congé payé avant et après l'accouchement. ${ }^{23}$

En raison du manque de familiarité avec le contexte politique yougoslave, les féministes en visite ont généralement eu du mal à comprendre la tentative de leurs hôtes d'articuler une critique féministe de gauche des aspects patriarcaux de l'autogestion yougoslave sans remettre en question ses postulats fondamentaux.

Nous ne critiquions pas le socialisme autogestionnaire yougoslave en tant que tel. Nous critiquions les éléments sexistes du système yougoslave, auquel nous nous identifiions en général. En ce sens, ce n'était pas une critique radicale du socialisme yougoslave... et quand vous regardez tous ces gens, ils étaient tous alors opposés au nationalisme, et ils le sont tous restés - je ne pense pas qu'il y ait une seule exception. $^{24}$

La tension entre hôtes et invités a atteint son paroxysme après certaines interventions masculines, en particulier celle du sociologue (croate/yougoslave) Slobodan Drakulić, qui a irrité les participantes étrangères au point que Christine Delphy, militante féministe lesbienne française et universitaire, l'a qualifié de « véritable caricature d'un discours masculin ${ }^{25}$ ». Une participante allemande a fini par s'emporter alors que Drakulić appelait à la patience et continuait son discours, entraînant une réaction encore plus forte de la partie internationale du public : «Assez! Basta! Enough! Dans quelle langue faut-il vous le dire ? ${ }^{26} »$. La mémoire autour de cet événement n'est pas univoque mais, selon Lepa Mlađenović, trente ans plus tard, c'est bien Christine Delphy qui a demandé à Drakulić de s'arrêter :

Pour moi, le tournant a été Christine Delphy, lorsque Delphy a dit à un homme : " Tu peux partir, tu t'arrêtes maintenant ». Il y avait quelques hommes participants qui voulaient être plus intelligents que nous. Il s'agissait d'un jeune sociologue de gauche. Il voulait nous dire... il a continué encore et encore, et Christine a juste dit «tu sais, tu t'arrêtes maintenant », ou «tu peux même partir »... quelque chose 
comme ça. C'était un énorme scandale ! Le quotidien Politika a écrit : « les féministes ont jeté les hommes hors de la conférence "! !7

Dans le compte-rendu de la conférence qu'elle a écrit dès son retour en France, Delphy ne mentionne pas le rôle central qu'elle aurait soi-disant joué pour faire taire Drakulić, mais elle précise que « dans cette agitation, la directrice du Centre a pris le micro pour protester contre une telle attitude 'discriminatoire', ajoutant avec colère que 'nous n'allons de toute façon pas faire de distinctions ici entre hommes et femmes' ${ }^{28}$, rencontrant l'approbation des autres participants yougoslaves. Sans remettre en cause la nécessité d'interrompre ce qui aurait pu être un discours masculin, je voudrais souligner comment l'insistance des organisateurs à ce qu'il n'y ait « aucune distinction entre hommes et femmes » reflétait l'effort socialiste pour universaliser (plutôt que de particulariser) l'expérience des femmes en l'abordant comme une question sociale d'intérêt général. Cette ligne de pensée a pu être suivie depuis la dissolution, en 1953, du Front antifasciste des femmes (Antifašistički front žena) - dissolution qui est parfois interprétée comme la conquête patriarcale ultime qui a mis un terme au processus d'émancipation des femmes en Yougoslavie. Vida Tomšič, un communiste, partisan et activiste slovène bien connu, a déclaré que la dissolution du Front avait été décidée parce que l'on considérait :

que son existence comme seule et unique organisation de femmes, structurée de manière totalement égalitaire, [...] aurait eu tendance à empêcher les femmes de participer aux efforts communs pour résoudre les problèmes sociaux et confortait l'idée fausse que le statut des femmes serait une question féminine distincte, et non une question touchant l'ensemble de la communauté et des combattants du socialisme...29

20 Si la conférence de 1978 a révélé des différences dans la manière dont la «question féminine " s'articulait avec le socialisme yougoslave et avec le capitalisme d'Europe occidentale, et si elle a constitué le moment fondateur du mouvement féministe yougoslave de l'après Seconde Guerre mondiale (générant une série d'initiatives plus ou moins formelles à la fois en Serbie et en Croatie, par exemple le groupe « Femme et société ", d'abord à Zagreb puis à Belgrade), elle est également devenue - peut-être par hasard - l'un des multiples points de départ de la mobilisation des militantes lesbiennes serbes/yougoslaves. La rencontre entre Christine Delphy et Lepa Mlađenović, « le bébé de la conférence », qui n'avait que 24 ans à l'époque, a eu un impact transformateur sur cette dernière. Mlađenović se souvient :

[J'essayais] de la comprendre. Elle était totalement différente de tous ceux qui vivent dans ma rue, c'est le but. Elle était totalement différente [...] d'où vient cette femme, de quel monde? Je n'en étais pas encore vraiment consciente... mais quelque chose m'attirait... elle n'était pas mariée. Elle marchait dans les rues sans faire attention aux yeux des hommes. Cette différence, je l'ai identifiée plus tard. ${ }^{30}$

21 La «différence » que Lepa Mlađenović a observée chez Christine Delphy était évidente dans la façon dont elle se comportait dans les restaurants où les participants se réunissaient après les temps officiels de la conférence.

Si je voulais choisir à qui je ressemblerais, je dirais à elle. Pas à cause de ce qu'elle disait théoriquement. Cette légèreté, j'avais l'impression qu'elle avait, dans un monde masculin, une démarche singulière; elle était autonome, indépendante, elle ne dépendait d'aucune validation hétérosexuelle pour entrer dans quelque lieu que ce soit, alors que chaque espace était un espace misogyne. J'ai senti qu'elle pouvait s'approprier chaque espace. Et j'ai vu en elle une énergie... elle ne s'attendait pas à ce que les serveurs du restaurant nous reconnaissent vraiment, elle n'avait pas l'intention d'expliquer à tous ces hommes qui nous sommes. En général, les femmes 
autour de moi essayaient de s'excuser ou de faire la cour aux hommes... elle, elle n'y consacrait aucune énergie... on n'a pas à y consacrer de l'énergie (...), c'est votre monde, même si vous savez que ce n'est pas le cas. En ce sens, elle a été un modèle crucial. Elle était intelligente, elle avait déjà intériorisé les principes féministes, et c'est ce que je sentais vraiment, elle montrait comment être à la hauteur des principes féministes.

Cette rencontre a été un point crucial dans la généalogie des mobilisations lesbiennes yougoslaves et aura des effets profonds au cours des décennies suivantes, car Lepa Mlađenović deviendra pour de nombreuses lesbiennes et féministes (post-)yougoslaves la figure inspiratrice que Christine Delphy a été pour elle.

En pratique, ce fut si intense pour moi et pour beaucoup d'entre nous que ce ne fut plus jamais la même chose, pour moi aussi. Pour moi, c'était totalement nouveau, que nous nous prenions au sérieux dans notre vie quotidienne, notre vie privée et en théorie. Cela a totalement changé la façon dont je me voyais dans la vie quotidienne et dont je voyais ce que je pouvais faire. Si je voulais faire des recherches, je pouvais me consacrer sérieusement à des études sur les femmes, je n'y avais jamais pensé auparavant. Et toute l'histoire de l'organisation en Yougoslavie a vraiment commencé après cette rencontre..$^{31}$

Dans les années à venir, Lepa Mlađenović allait devenir le visage le plus reconnaissable de l'activisme lesbien féministe (post-)yougoslave. Véritable militante yougoslave, agent de liaison dans le sens où elle couvrait l'ensemble du territoire de l'ancien État et étendait son influence vers d'autres parties de l'Europe et des États-Unis, Mlađenović a inlassablement rassemblé et autonomisé les femmes tout en promouvant la politique de séparation femmes/lesbiennes. En 1986, Mlađenović a plaidé en faveur d'activités réservées aux femmes au sein du groupe de Belgrade «Femme et société ${ }^{32}$; en 1993, elle a cofondé le Centre autonome des femmes de Belgrade (Autonomni ženski centar) ; et, en 1995, elle a contribué à la création de la première organisation lesbienne "Labris ", née d'une séparation avec le premier groupe militant serbe non hétérosexuel "Arkadija». Elle a également encouragé de nombreuses initiatives d'activistes lesbiennes qui ont eu lieu dans la région. Comme l'écrit Irena Cvetkovic, une militante de Macédoine du Nord :

le groupe de lesbiennes [...] a commencé à organiser des ateliers et des événements sur différents sujets, principalement en collaboration avec des Serbes et des activistes d'autres anciennes républiques yougoslaves, comme Lepa Mlađenović. Lepa s'est rendue assez souvent à Skopje pour soutenir ses sœurs macédoniennes et a insisté pour que les femmes lesbiennes se séparent des organisations LGBT générales. ${ }^{33}$

24 C'est donc principalement à travers la figure de Mlađenović que le séparatisme femmes/lesbiennes a pu être retracé à travers de nombreux épisodes d'engagement féministe (post-)yougoslave, culminant avec la Marche des lesbiennes de 2015.

\section{La Marche lesbienne de Belgrade en 2015}

La Marche lesbienne de Belgrade s'est déroulée dans le cadre du Printemps lesbien, une manifestation de quatre jours, organisée en avril 2015 par un groupe d'activistes de Serbie et de Bosnie-Herzégovine. Elle a réuni des lesbiennes des régions exyougoslaves, ainsi que leurs amis et soutiens à l'international associés à la Caravane féministe qui a traversé l'Europe entre mars et octobre 2015, s'arrêtant à Belgrade et à Zagreb, dans le but de documenter les luttes des femmes pour l'égalité. Le programme 
du Printemps lesbien était composé d'un ensemble d'ateliers et de panels et a été clôturé par la Marche lesbienne. Zoe Gudović, une activiste lesbienne et une des organisatrices du Printemps lesbien, a affirmé que le principal objectif de la Marche était d'attirer l'attention du public sur la discrimination à laquelle font face les femmes et les lesbiennes dans leur vie quotidienne :

En descendant dans la rue, nous montrons que nous n'avons pas été importées de l'Ouest, que nous existons dans ce pays, que nous sommes des citoyennes, que nous construisons cette société et que nous nous efforçons de la faire s'habituer aux changements. De tels changements se produiront lorsque les gens comprendront que l'oppression politique des femmes et des lesbiennes en particulier est omniprésente. Aujourd'hui nous sommes sorties de ce cadre oppressif, nous avons dit que nous ne voulions pas faire partie d'un système qui nous exploite: nous sommes conscientes qu'en agissant ainsi nous sommes devenues une menace. Avec cet événement aujourd'hui et avec l'ensemble du programme [du Printemps lesbien], nous avons montré notre force et le fait que nous sommes totalement capables et prêtes à changer les conditions sociales. Ce n'est, bien entendu, pas facile, mais c'est à nous de parcourir le chemin. ${ }^{34}$

La Marche a également été vue comme une opportunité de s'engager dans une forme alternative d'activisme qui constituerait une critique implicite des initiatives gay et gauchistes existant en Serbie et dans la région, lesquelles sont perçues comme étant trop patriarcales et centrées sur les hommes. Ana Pandej, l'une des organisatrices du Printemps lesbien, affirmait ainsi :

La Marche lesbienne représente le point culminant du programme du Printemps lesbien que nous avons organisé car dans notre société les lesbiennes sont rendues invisibles de plusieurs façons. Nous sommes ici, nous participons aux différentes mobilisations, mais ce n'est pas reconnu. Nous participons aux manifestations des travailleurs, à celles des femmes, à la Pride... Mais il n'y a jamais de lesbiennes, ce sont toujours d'autres groupes qui occupent les premières lignes. Il est extrêmement important que les femmes en général et surtout les lesbiennes, suivies par d'autres camarades femmes, y compris hétérosexuelles, bisexuelles, ou queer, revendiquent par elles-mêmes l'espace public. ${ }^{35}$

27 À cet égard, une des annonces de la Marche fait référence au caractère volontaire de l'engagement des organisatrices, présentant ainsi l'événement comme étant aux antipodes des formes d'activisme professionnalisé dans la région, qui ont engendré un éloignement considérable entre les activistes et celles au nom desquelles ces mêmes activistes sont censées agir ${ }^{36}$. Les organisatrices ont déclaré :

Nous sommes un groupe de lesbiennes locales, d'amies, d'activistes de différents groupes de Serbie et de Bosnie-Herzégovine et nous voulons que le Printemps lesbien fleurisse en ce mois d'avril! Notre travail est fondé sur des principes féministes et il se fait sur la base du volontariat. Notre intention est de nous dresser sur le chemin du patriarcat et de la lesbophobie, et de marquer les 25 ans du mouvement lesbien en Serbie! Nous sommes toutes conscientes que les conditions sociales sont extrêmement défavorables pour les droits des lesbiennes - nous faisons face à une lesbophobie permanente, les attaques contre les lesbiennes sont fréquentes et il est potentiellement dangereux de se tenir par la main dans des lieux publics ou de montrer de l'affection. ${ }^{37}$

La Marche s'est tenue le 19 avril. Elle a démarré au Parc des pionniers (Pionirski park), a traversé les rues Kralja Milana et Resavska et s'est terminée au Centre de décontamination culturelle (Centar za kulturnu dekontaminaciju), probablement le lieu clé de la "scène " alternative de Belgrade, étroitement associé à l'initiative anti-guerre d'intellectuels serbes rassemblés sous le nom de «Cercle de Belgrade» (Beogradski 
krug). Une vidéo de la Marche, réalisée par Myriam Fougère ${ }^{38}$, montre un groupe de femmes qui, encouragées par Zoe Gudović, chantent le slogan «Nous sommes toutes lesbiennes et nous en sommes fières!» (Lezbejke smo sve zato ponosne smo bre!). Ce slogan était l'élément central d'un chant qui comprenait aussi les paroles suivantes :

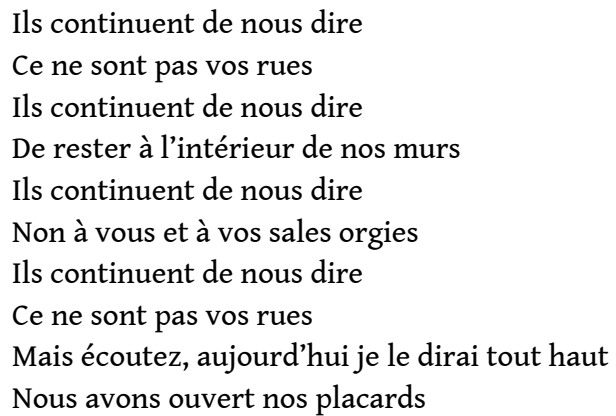

Entonner le chant de Gudović a été l'un des moments les plus marquants de la Marche, car les participantes ont pu exprimer leur sentiment de vivre dans un environnement social profondément lesbophobe, tout en transformant l'événement en un moment d'émancipation qui leur a permis de réaliser un coming out collectif. Cet acte a constitué une expression explicite de prise de parole lesbienne ; la "sortie du placard» a été formulée de manière claire et même rebelle ("écoutez, aujourd'hui je le dirai tout haut »), remplissant les rues de Belgrade et la sphère publique serbe d'une présence lesbienne jusque-là sans précédent.

L'une des banderoles les plus en vue portées par les activistes proclamait "Les lesbiennes soutiennent les lesbiennes» (Lezbijke uz lezbejke - Hr $\bullet \mathrm{Sr}$ ). Le premier mot est écrit en croate, le deuxième en serbe. L'agentivité lesbienne est ainsi apparue comme une force antinationaliste, opposée à la violence, mettant en lumière les liens entre activistes de ces deux pays post-yougoslaves, engagés dans un conflit armé dans les années 1990 et qui ont conservé depuis des relations très tendues. La force de l'effet produit par cette banderole résidait dans le fait de mettre en avant plus d'une décennie de résilience des liens féministes serbo-croates, de souligner la capacité du féminisme à contourner les régimes produisant de l'homogénéité et de continuer de faire de la région un espace de lutte politique commune. La Marche a eu un caractère non seulement régional mais a également été, plus largement, transnationale, car les slogans chantés et les banderoles écrites utilisaient plusieurs langues (arabe, albanais, français, espagnol, entre autres), mettant ainsi l'accent sur le besoin d'une solidarité internationale des femmes et des lesbiennes.

31 Pourtant, à côté de ces caractéristiques positives, l'organisation de la Marche a, dès le départ, été accompagnée de tensions. La première annonce envoyée au groupe razotkvirivanje afin d'encourager la participation affirmait que la « Marche est lesbienne et donc uniquement à l'intention des femmes. La Marche célèbre le mouvement lesbien en Serbie et promeut des politiques féministes ${ }^{39}$ ». Cette partie de l'invitation des organisatrices a donné lieu à un débat parmi les membres du groupe, au cours duquel l'activiste gay serbe Dušan Maljković a déclaré :

C'est incroyable de voir qu'une manifestation politique organisée en 2015 exclut un sexe. Ma première réaction a été la surprise et l'incrédulité. Pourquoi agir ainsi ? J'ai essayé de trouver une réponse [dans la phrase qui déclare que la Marche est lesbienne]. Mais ce type d'argument (absent sur le fond) va apparemment de soi, alors qu'il est fondamentalement contradictoire. Si la Marche est lesbienne, alors pourquoi envoyer l'invitation à toutes les femmes et donc aussi à des non- 
lesbiennes? J'aurais compris si cela avait été un événement privé ou une sorte d'atelier psychologique dans le cadre d'une manifestation publique. Mais le fait de conseiller à quelqu'un de ne pas participer [à une manifestation] dans l'espace public en raison de son sexe est pour moi un acte scandaleusement répressif. Je ne souhaite même pas commenter à quel point il est contreproductif politiquement d'exclure les sympathisants masculins du mouvement lesbien; de telles personnes existent et je me considère comme l'une d'entre elles. ${ }^{40}$

32 La décision des organisatrices d'exclure les hommes est également frappante car les manifestantes ont dû se laisser entourer par un cordon de policiers, qui les a accompagnées tout le long du trajet de la Marche. L'invitation précisait que les organisatrices avaient déclaré la Marche auprès de la police, mais n'en avaient pas informé les médias. Les activistes LGBT ont parfois recours à cette stratégie afin de ne pas laisser suffisamment de temps aux membres de groupes d'extrême-droite de mettre en place une mobilisation potentiellement agressive, réduisant ainsi la probabilité que des violences se produisent. Le cordon de policiers (devenu typique pour certaines manifestations "impopulaires » comme celles des «Femmes en noir » [Žene u crnom]) revient à créer de fait une membrane qui sépare les activistes du public. Si cette stratégie assure la sécurité, elle met au grand jour la tension entre les participantes à la Marche et ses observateurs, car elle produit une « enclave activiste » qui tend à rendre la manifestation exotique et brouille le sens de public dans la notion d'espace public. En contradiction avec l'intention d'organiser une manifestation sans hommes, les marcheuses sont restées enfermées dans le paradigme patriarcal car elles ont dû être protégées par une force de police d'État, majoritairement masculine qui, au cours des décennies passées, a été associée à des violences infligées aux personnes LGBT et aux dissidents politiques.

33 Alors même que la Marche a eu une importance symbolique immense, sa forme, contrainte par une forte présence policière, a limité sa capacité d'être plus qu'un incident et de devenir une fissure ou un moment non hétéronormatif qui aurait pu se diffuser en direction de ceux qui n'étaient pas explicitement invités ou personnellement intéressés par la cause. Ne pas annoncer la manifestation et donc limiter sa dimension publique est une décision compréhensible quand on veut éviter la violence. Mais il a fallu en payer le prix fort, car cette décision a eu pour effet de réduire la participation à la Marche aux cercles étroits des organisatrices, ce qui a mis en lumière le caractère spécifiquement urbain de cet activisme, comme le révèlent les propos critiques d'une participante dans le débat au sein du groupe razotkvirivanje :

Les féministes radicales sont des fascistes. Ce n'est pas une blague. C'est une forme de fascisme et peu importe si cela a lieu dans les rues de San Francisco ou dans les rues de Belgrade. Dans tous les cas, qui va à ces marches de la fierté ici, sinon celles qui sont dans une position très privilégiée? Celles qui participent à de tels événements sont des gens qui habitent à 100 mètres de ce parc [le Parc des pionniers à Belgrade] et qui ont tous les privilèges. $99 \%$ des hommes de ce pays subissent plus de discriminations et sont dans une situation bien pire que n'importe quelle séparatiste qui participe à la Marche ou à la soi-disant parade de la soi-disant fierté. $^{41}$

Les principales orientations des initiatives d'activistes lesbiennes (et gay) en Serbie se sont développées dans le cadre des mouvements anti-guerre au cours des années 1990, avec la mobilisation de « ces représentants d'une identité culturelle répandue, partagée en Yougoslavie, urbaine, cosmopolite et fondamentalement non ethno-nationaliste, qui s'exprimaient le mieux ${ }^{42} »$. L'activisme civique est apparu à l'époque également comme 
une stratégie permettant de continuer de faire partie d'une classe moyenne urbaine, tandis que cette dernière était alors en train de disparaitre sous le poids de l'appauvrissement et de l'isolement international ${ }^{43}$. De nombreux activistes se sont embarqués dans le processus d'ONG-isation, ce qui signifie que les initiatives locales ont été remplacées par des "projets" dont la mise en œuvre comportait la gestion de budgets et la participation aux aspects financiers, ce qui s'est révélé être une source de tension et de frustration. Même si les organisatrices de la Marche ont revendiqué leur « indépendance » et ont réalisé le travail organisationnel sur la base du volontariat, en regardant la liste des organisations qui ont soutenu l'événement, on constate que la majorité d'entre elles sont, d'une manière ou d'une autre, liées à des ONG de défense des droits de l'Homme basées à Belgrade (dont certaines gèrent des sommes d'argent considérables et jouent le rôle de gardiennes de l'accès aux projets orientés vers les femmes, dans la mesure où elles décident de ce qui sera ou ne sera pas financé). La division du travail et la compétition pour des ressources limitées génèrent des clivages entre les organisations, clivages qui mènent à dupliquer des initiatives, comme des "premiers» événements à répétition dont les milieux non urbains se trouvent écartés ${ }^{44}$. Comme l'a affirmé un activiste :

L'objectif de la Marche n'était pas du tout clair car, tout d'abord, très peu de femmes étaient au courant. Il semble que l'ensemble de l'événement a été organisé pour les organisatrices elles-mêmes, comme elles n'ont pas fait l'effort d'informer les femmes qui ne sont pas liées à leurs organisations. Une telle décision a eu un effet particulièrement néfaste sur les femmes lesbiennes vivant dans les villes de province, car cela a été une occasion ratée de leur montrer qu'elles ne sont pas seules. $^{45}$

Larisa Kurtović écrit que dans le contexte de Sarajevo assiégée, l'urbanité était synonyme d'idéaux humanistes et cosmopolites, méfiants à l'égard des politiques institutionnelles et insistant sur l'expression créative en contrepoint du « patriotisme, [de] la fierté nationale ou [de] la tradition ${ }^{46} »$. Une telle « exclusivité urbano-centrée $»^{47}$ a conduit à la conviction qu'un habitus urbain partagé par les anciennes républiques yougoslaves avait remplacé les affiliations nationales en créant un sentiment de proximité entre les "urbains", plutôt qu'entre ces derniers et leurs co-nationaux " moins urbains » ou ruraux (même s'il peut également exister des hiérarchies au sein de ces catégories). Alors que des femmes de petites villes serbes ont pris part aux activités du Printemps lesbien, il semble que plus de trois décennies d'engagement féministe en Serbie n'ont pas ébranlé l'idée que les luttes d'émancipation sont un phénomène urbain réservé aux femmes des classes moyennes ${ }^{48}$. La Marche des femmes contre le fascisme de $2017 \mathrm{a}$ eu lieu à Belgrade et a également démarré au Parc des pionniers, réunissant probablement de nombreuses activistes ayant participé à la Marche lesbienne. Comme l'écrit Savić à propos de cette marche, qu'elle appelle un « reality show de la solidarité »:

Embrasser le féminisme en Serbie est un privilège de classe de femmes blanches et éduquées, majoritairement de Belgrade. C'est une chose difficile à faire lorsque l'on est pauvre ou que l'on travaille à la National Cash Register ${ }^{49}$ - ce qui est déjà un privilège en soi - [...], une entreprise qui exploite [...], où l'on fait des journées et des nuits de travail hallucinantes et interminables, qui pompent l'énergie à tel point qu'on n'a plus la force de regarder des lettres écrites sur un bout de papier [...] sans parler d'utiliser un ordinateur pour s'informer de ce qui se passe autour. [...] Le féminisme ne s'est jamais montré spécialement intéressé d'inclure les femmes employées par la NCR, ne parlons même pas d'autres femmes moins « chanceuses ». Il ne propose aucune solution aux femmes de la NCR. [...] [De telles 
femmes] ne s'intéressent pas au féminisme académique gauchiste des femmes blanches. Ce que Althusser a dit pourrait peut-être leur être utile, mais elles n'ont pas la force ou le temps de le lire, d'apprendre que cela existe..$^{50}$ activiste policée, renforce le sentiment des activistes d'être à part, différentes du public plus large auquel elles s'adressent dans le but de créer un changement social. À cet égard, les activistes apparaissent comme des actrices «intermestiques" (internationales et domestiques), qui traduisent les valeurs universelles de la modernité occidentale en programmes localement réalisables - une tâche qui ne peut être effectuée sans recréer des hiérarchies imbriquées ${ }^{51}$. C'est aussi la raison pour laquelle, dans le débat en ligne, certains participants ont pointé le fait d'avoir "été informés " de la Marche comme s'agissant d'un fait accompli, plutôt que d'avoir été invités à prendre part à la discussion sur la manière d'organiser l'événement. Comme l'a déclaré une militante,

[La Marche lesbienne] montre que l'activisme LGBT, y compris sa composante lesbienne, échoue sans cesse à l'examen du féminisme et de la prise de décision démocratique. La question n'est pas tant de savoir si vous m'informez en tant que lesbienne en faisant passer un message du sommet vers la base que l'événement sera réservé aux femmes (même pas aux lesbiennes), mais que vous devriez nous demander si nous toutes (la majorité) au sein de la communauté sommes d'accord avec la politique que vous êtes en train de mettre en place. Ce dont il est question ici est un événement public politique et non pas une fête privée ou un sexe club [...] au moins cent d'entre nous ne sont pas venues à cause de cette politique. ${ }^{52}$

Cet extrait du débat en ligne met en lumière les difficultés qu'il y a à conceptualiser et à pratiquer une politique annonciatrice de transformation dans des environnements autoritaires et appauvris. Le style de gouvernance qui met au centre un leader est souvent repris et approprié au sein des cercles militants. Il en résulte une absence de dialogue sensé et le rejet facile d'opinions alternatives et dissidentes.

De plus, la Marche a révélé le paradoxe inhérent au séparatisme lesbien féministe radical à travers la reproduction et l'essentialisation du paradigme binaire du genre dans l'espace public. Ces marches lesbiennes organisées, comme celle de Belgrade, "seulement pour les femmes" ne peuvent éviter l'écueil de définir, au moins implicitement, qui peut être défini comme femme. Tandis que, d'un côté, le féminisme radical combat les impositions "patriarcales" concernant l'apparence des femmes, il peut, d'un autre côté, finir par adopter les définitions les plus répandues de la féminité. Comme l'a formulé Maljković,

La Marche lesbienne est une manifestation exclusive qui, pour quelques heures, dans un espace délimité, met en scène une dimension traditionnellement patriarcale du pouvoir, qui est une exclusion sexuelle; elle ne fait qu'inverser les places, le sexe inclus étant celui qui est habituellement exclu dans la société englobante, sans rien changer à la forme du pouvoir qui se donne à voir ${ }^{53}$. Elle transforme l'espace public en espace privé, alors qu'une manifestation inclusive aurait en réalité déconstruit le pouvoir patriarcal en ouvrant l'espace pour construire une égalité politique, en reconnaissant l'universalité et le caractère démocratique de l'espace public ${ }^{54}$.

39 En fin de compte, la Marche a apporté de la division et de nombreuses femmes n'y ont pas participé. Comme l'a souligné Lepa Mlađenović dans l'échange en ligne consécutif à l'événement :

Il a manqué une discussion dans la communauté au sens large, l'information et les clarifications fournies par les organisatrices ont été insuffisantes, il n'y a eu ni 
négociation ni accord [...]. J'ai compris qu'il y a eu de nombreuses erreurs d'organisation. C'est la raison pour laquelle nombreux sont les activistes qui se sentent blessés et je le regrette profondément. ${ }^{55}$

\section{Conclusion}

Organiser une marche internationale lesbienne à Belgrade a été une grande réussite militante. Jamais auparavant les rues de la capitale serbe n'avaient connu manifestation aussi prononcée de l'agentivité lesbienne, repoussant les frontières de la citoyenneté sexuelle normative et lesbophobe. La Marche, en tant que manifestation collective, a mis en évidence la fragilité des avancées émancipatrices à l'époque de la montée du populisme. Elle a souligné la persévérance des attitudes patriarcales non seulement dans la population en général, mais aussi au sein des initiatives militantes qui visent à la libération (non hétéro)sexuelle. Cependant, en interdisant effectivement la participation des hommes, la Marche est devenue un double symptôme de ce retour intense du patriarcat: d'une part, par sa forme, basée sur une binarité stricte de genre, qui a inversé les rôles (les femmes excluant les hommes de l'espace public) sans déstabiliser, mais plutôt en reproduisant le mécanisme patriarcal d'exclusion; d'autre part, par son contenu, idéologique et affectif, découlant de la marginalisation continue des droits des femmes. Par conséquent, la forme de l'événement a été un reflet éloquent du grief qui l'a rendu possible.

41 En excluant explicitement les hommes de leur rassemblement, les organisatrices de la Marche ont porté atteinte à la nature publique de l'espace public, ainsi qu'à la fonction contestataire des contre-publics subalternes ${ }^{56}$. Bien qu'ils puissent involontairement se retrouver enclavés, dans la mesure où ils souhaitent être publics et revendiquer l'espace public, ceux-ci ne peuvent se présenter comme des "enclaves ». En d'autres termes, les contre-publics ont en principe tendance à militer contre le séparatisme parce qu'ils adoptent une modalité orientée vers le public qui leur permet de se construire en tant que membre d'un public et d'interagir de manière discursive avec d'autres acteurs de l'espace public. Cela signifie que, aussi subalternes qu'ils puissent être, les membres d'un contre-public se perçoivent comme faisant partie d'un public potentiellement plus large auquel ils s'adressent et qu'ils veulent influencer.

Cette stratégie d'exclusion a également fait redécouvrir aux organisatrices de la Marche le paradoxe qui est au cœur de l'organisation de nombreuses organisations séparatistes lesbiennes : alors que les activistes ont tendance à adopter de manière déclarative une idéologie d'inclusion, la marche finit par servir de véhicule pour célébrer une identité beaucoup plus étroite qui ne peut être partagée par toutes les femmes (non hétérosexuelles) ou d'autres partisans potentiels de la cause non hétérosexuelle. Même si la plupart des marches lesbiennes contemporaines, en tant qu'événements publics de plus longue tradition, incluent les personnes trans (par exemple, la Los Angeles Lesbian Love March), les marches qui excluent «l'autre sexe » ou les groupes transgressant les règles de genre ont tendance à perpétuer le système binaire de genre qui constitue le noyau de l'oppression basée sur le genre.

Toute initiative militante liée à l'identité se heurte à ce que Gamson appelle le « dilemme queer » : les catégories d'identité fixes sont à la fois la base de l'oppression et du pouvoir politique ${ }^{57}$; si le fait de saper des identités particulières peut perpétuer la discrimination et être politiquement préjudiciable, le fait de les rendre essentielles 
peut contribuer à favoriser les conditions structurelles qui rendent cette discrimination possible. Les militants sont souvent enfermés dans une impasse identitaire à laquelle il est difficile d'échapper. Le fait que certaines des organisatrices de la Marche lesbienne (comme, par exemple, Zoe Gudović) aient participé, il y a des années, à l'expérimentation de la notion de queer au sein du "Collectif queer de Belgrade" montre que la queerness (qui pose le problème de la viabilité des identités sexuelles ou de genres fixes) n'a pas tenu ses promesses politiques dans la région post-yougoslave. Les initiatives LGBT sont plutôt aux prises avec des boucles temporelles dans lesquelles des approches plus essentialisantes coexistent (et parfois entrent en concurrence) avec d'autres désessentialisantes. Cela montre que la question de comment stabiliser temporairement et stratégiquement les identités doit être soulevée - encore et encore de manière à garantir que les luttes fondées sur celles-ci ne finissent pas par affaiblir la cause de la libération sexuelle.

Bien que les négociations de ce type exigent un travail politique à long terme, une résolution radicale du dilemme queer qui consisterait à exclure des individus des événements se déroulant dans les espaces publics ne peut transformer l'enthousiasme des participantes en des répertoires existentiels plus durables et plus largement mobilisables. Par un tel choix stratégique, qui favorise une binarité stricte de genre, l' «enclave des activistes » devient non seulement physique, délimitée par un cordon policier, mais aussi identitaire. De la même manière qu'une enclave physique met à nu la tension entre les participantes et le public observateur, l'enclave identitaire constitue une boîte aux frontières contrôlées, qui implose nécessairement (par le biais de luttes intestines) car elle n'est jamais assez large pour contenir la variabilité de l'expérience des femmes et des humains en général.

Dans cette optique, la Marche a fourni une illustration frappante des processus par lesquels le savoir féministe a été constamment évacué après le socialisme yougoslave. Dans cet article, je suis revenu sur la conférence de 1978 "Camarade femme», qui a constitué l'un des points cruciaux de l'organisation féministe dans l'espace yougoslave, pour démontrer que l'héritage progressiste du régime socialiste, que les participantes à la conférence yougoslave ont à la fois critiqué et reconnu, n'a pas été mobilisé pour contrer la décision des organisatrices de la Marche d'empêcher les hommes de participer à un événement public. Les militantes ne se sont pas appuyées sur leurs prédécesseures socialistes pour remettre en question une telle option stratégique du point de vue des luttes humaines universelles qui se déroulent dans l'espace public - il n'y avait pas de féministe socialiste pour dire, comme beaucoup l'ont fait près de quatre décennies auparavant, qu'un tel choix stratégique constituerait non seulement un acte de discrimination mais qu'il s'avérerait finalement autodestructeur.

Dans le cadre de la Marche, la réification de la binarité de genre, qui constitue le noyau structurel de la distinction entre les sexes, était une expression de la politique identitaire étroitement associée à la domination capitaliste néolibérale. D'un côté, la binarité de genre a été problématisée dès les premiers stades du socialisme yougoslave par l'effort de mise sur un pied d'égalité constitutionnelle du statut politique, social et économique des hommes et des femmes (ce qui a déstabilisé mais n'a pas éradiqué un patriarcat profondément enraciné); d'un autre côté, elle est une caractéristique de base du capitalisme moderne, crucial pour la manière dont il organise la division du travail. C'est pour cette raison que l'expansion capitaliste effrénée et le retour au patriarcat généralisé se sont déroulés "main dans la main " après la chute du 
socialisme: le capitalisme s'efforce de réduire le coût du travail en reproduisant systématiquement le patriarcat et en dévalorisant les droits des femmes, en les maintenant dans une position de dépendance vis-à-vis des hommes, tout en n'accordant qu'à certains le privilège de bénéficier des avantages de l'ordre libéral ${ }^{58}$.

Les lesbiennes étant particulièrement menacées lorsque le statut des femmes est en déclin, il n'est pas étonnant qu'une manifestation lesbienne exclusivement féminine ait signalé la nature systémique et multidimensionnelle du changement social qui s'est produit dans la région yougoslave au cours des trois dernières décennies. Cette transition douloureuse a intégré l'espace post-yougoslave dans le « temps de séquence occidental $\aleph^{59}$ qui aplatit les temporalités précapitalistes et éclipse les acquis socialistes. Une telle pratique invisibilise les réalisations des générations précédentes et place les militantes dans la "deuxième vague féministe ", leur insufflant le sentiment qu'elles sont pionnières, car elles sont aujourd'hui contraintes de s'engager et de reprendre des luttes qui avaient été, dans une large mesure, gagnées il y a des décennies.

Toutefois, le vide théorique créé par la dissolution de la Yougoslavie et par l'affaiblissement de ses traditions féministes socialistes n'aurait pas pu être comblé aussi rapidement par les connaissances et les stratégies féministes développées dans les pays occidentaux du capitalisme avancé (par exemple, les marches des fiertés, les marches lesbiennes, etc.) s'il n'y avait pas eu de préoccupation majeure pour le patriarcat tant dans le système capitaliste que socialiste ${ }^{60}$. La région post-yougoslave n'a pas seulement été « envahie » par les idéologies séparatistes (lesbiennes) qui ont fleuri aux États-Unis tout au long des années 1970 - un tel argument ferait l'impasse sur l'agentivité des acteurs locaux. Au contraire, les options idéologiques séparatistes comptaient sur les courants activistes locaux qui, au cours des dernières décennies, sont devenus plus ou moins visibles. Dans le sillage du socialisme, ils ont pu réapparaître avec une radicalité que ces préférences idéologiques avaient largement perdue dans leurs lieux d'origine : l'intensification du militantisme trans a entre-temps dévoilé leurs dimensions discriminatoires ouvrant - une fois de plus - la voie à la politique progressiste adoptée par tous ceux qui sont favorables à l'égalité des genres. En ce sens, la Marche lesbienne de Belgrade de 2015 n'était pas simplement une manifestation "ponctuelle» organisée à la hâte par un groupe de "militantes indépendantes ». Elle a plutôt été un événement qui, en se déroulant dans l'espace public, a révélé la trajectoire tortueuse mais résistante d'un des volets de l'engagement féministe (post-)yougoslave dont les effets en termes d'enthousiasme, d'autonomisation et de liberté sont tempérés par les douleurs liées à la division et la discrimination.

\section{NOTES}

1. BILIĆ Bojan, RADOMAN Marija (dir.), Lesbian Activism in the (Post-)Yugoslav Space: Sisterhood and Unity, Londres, Palgrave Macmillan, 2019.

2. Ce groupe virtuel de conversation rassemble des militant.e.s LGBT principalement de Serbie et de l'espace yougoslave. Son nom est un néologisme qui combine le mot razotkrivanje qui signifie 
dévoiler, découvrir et kvir - queer. Il y a eu 52 posts dans ce groupe à la suite de l'annonce de la Marche.

3. Clarke John, Bainton Dave, LendVai Noémi, stubbs Paul, Making Policy Move: Towards a Politics of Translation and Assemblage, Bristol, Policy Press, 2015.

4. DELLA PORTA Donatella, «Protests as Critical Junctures: Some Reflections Towards a Momentous Approach to Social Movements ", Social Movement Studies, vol. 19, n 5-6, 2020, p. 556-575.

5. FRASER Nancy, «Rethinking the Public Sphere: A Contribution to the Critique of Actually Existing Democracy », Social Text, vol. 25/26, 1990, p. 56-80.

6. Ibidem, p. 71

7. BILIĆ Bojan, STUBBS Paul, « Unsettling 'the Urban' in Post-Yugoslav Activisms: Right to the City and Pride Parades in Serbia and Croatia ", dans Kerstin JACOBSSON (dir.), Urban Movements and Grassroots Activism in Central and Eastern Europe, Londres, Ashgate, 2015, p. 119-138.

8. Podmore Julie, «Contested Dyke Rights to the City: Montréal's 2012 Dyke Marches in Time and Space ", dans Kathe BROWNE, Eduarda FEREIRA (dir.), Lesbian Geographies: Gender, Place and Power, Londres, Routledge, 2016, p. 71-89.

9. ENSZER Julie R., « How to Stop Choking to Death': Rethinking Lesbian Separatism as a Vibrant Political Theory and Feminist Practice ", Journal of Lesbian Studies, vol. 20, n² 2, 2016, p. 180-196 (181).

10. LEVY Ariel, «Lesbian Nation: When Gay Women Took to the Road », The New Yorker, 2009, en ligne : www.newyorker.com/magazine/2009/03/02/lesbian-nation (consulté en juin 2020).

11. Radicalesbians, «The Woman Identified Woman ", 1970, en ligne: https:// dukelibraries.contentdm.oclc.org (consulté en juin 2020).

12. Ibid.

13. RICH Adrienne, « Notes for a Magazine: What Does Separatism Mean? », Sinister Wisdom, $\mathrm{n}^{\circ} 18$, 1981, p. 83-91 (88).

14. MCCONNELL Elizabeth A. et al., "Trans Women and Michfest: An Ethnophenomenology of Attendees' Experiences ", Journal of Lesbian Studies, vol. 20, n 1, 2016, p. 8-28.

15. Combahee River Collective, «A black feminist statement», 1978, en ligne: https:// americanstudies.yale.edu (consulté en juin 2020). Ndlt: traduction inspirée de FALQUET Jules, « Déclaration du Combahee River Collective », Les cahiers du CEDREF, vol. 14, 2006, p. 53-67.

16. LEVY, « Lesbian Nation », art. cité.

17. TRIGILIO Jo, «Complicated and Messy Politics of Inclusion: Michfest and the Boston Dyke March ", Journal of Lesbian Studies, vol. 20, nº 2, 2016, p. 234-250.

18. BROWN-SARACINO Japonica, GHAZIANI Amin, "The Constraints of Culture: Evidence from the Chicago Dyke March », Cultural Sociology, vol. 3, nº 1, 2009, p. 51-75.

19. PODMORE, « Contested Dyke Rights », art. cité.

20. GHAZIANI Amin, FINE Gary A., «Infighting and Ideology: How Conflict Informs the Local Culture of the Chicago Dyke March ", International Journal of Politics, Culture and Society, $\mathrm{n}^{\circ} 20,2008$, p. 51-67.

21. Ces initiatives seront connues sous le nom de "nouveau féminisme " car elles marquent l'apparition d'un discours politique différent de celui qui s'inscrivait dans les institutions de l'État yougoslave (« féminisme d'État »).

22. Dragan Klaić, cité par BONFIGLIOLI Chiara, Belgrade 1978. Remembering the Conference "Drugarica zena. Zensko pitanje - novi pristup?" "Comrade woman. The women's question: A new approach?" thirty years after, Université d'Utrecht, 2008, p. 54.

23. Constitution of the Federative People's Republic of Yugoslavia, 1946, en ligne: https:// www.worldstatesmen.org/Yugoslavia_1946.txt (consulté en septembre 2020).

24. Dragan Klaić, cité par BONFIGLIOLI, Belgrade 1978, op. cit., p. 100. 
25. DELPHY Christine, « Nouvelles de Yougoslavie : lueurs féministes en pays socialistes (Belgrade 1978) », Questions féministes, nº 5, 1979, p. 129-136 (131).

26. Ibid., p. 131.

27. Lepa Mlađenović, cité par BoNFIGLIOLI, Belgrade 1978, op. cit., p. 92.

28. DELPHY, « Nouvelles de Yougoslavie », art. cité, p. 131.

29. томšı̌ Vida, Woman in the Development of Socialist Self-Managing Yugoslavia, Belgrade, Jugoslovenska stvarnost, 1980, p. 80.

30. Lepa Mlađenović, citée par BONFIGLIOLI, Belgrade 1978, op. cit., p. 91.

31. Ibid., p. 93.

32. LóRÁND Zsófia, The Feminist Challenge to the Socialist State in Yugoslavia, Londres, Palgrave Macmillan, 2018.

33. cVETKOVIC Irena, « Breaking the Silence: Lesbian Activism in Macedonia », dans Bilić, Radoman (dir.), Lesbian Activism, op. cit., p. 109-132 (124).

34. TELEGRAF, «Lezbejsko proleće: Održan prvi ženski gej marš u Beogradu, da li je protekao bez incidenata?» [Le printemps lesbien : La première marche des homosexuelles s'est tenue à Belgrade, s'est-elle passée sans incident ?], 2015, en ligne : www.telegraf.rs (consulté en juin 2020) 35. Ibid.

36. BUTTERfIELD Nicole, «Discontents of Professionalisation: Sexual Politics and Activism in Croatia in the Context of EU Accession », dans Bojan Bilić (dir.), LGBT Activism and Europeanisation in the Post-Yugoslav Space: On the Rainbow Way to Europe, Londres, Palgrave Macmillan, 2016.

37. Labris, « Najava: Lezbejsko proleće u Beogradu od 16. do 19. Aprila » [Annonce : le printemps lesbien à Belgrade du 16 au 19 avril], 2015, en ligne : http://arhiv2015sr.labris.org.rs/najavalezbejsko-prolece-u-beogradu-od-16-do-19-aprila/ (consulté en septembre 2020).

38. Video Lesbian March, 2015, en ligne : www.youtube.com/watch?v=QFJD3DwdP3c (consulté en juin 2020).

39. SANJA, Razotkvirivanje, mai 2015, en ligne: https://groups.google.com/g/razotkvirivanje (consulté en juin 2020).

40. мALjKović Dušan, Razotkvirivanje, mai 2015, en ligne: https://groups.google.com/g/ razotkvirivanje (consulté en juin 2020).

41. Aleksa, Razotkvirivanje, mai 2015, en ligne: https://groups.google.com/g/razotkvirivanje (consulté en juin 2020).

42. DEvić Ana, "Anti-War Initiatives and The Un-Making of Civic Identities in The Former Yugoslav Republics ", Journal of Historical Sociology, vol. 10, n 2, 1997, p. 127-156 (156).

43. BILIĆ Bojan, We Were Gasping for Air: (Post-)Yugoslav Anti-War Activism and Its Legacy, BadenBaden, Nomos, 2012.

44. Dans un débat en ligne sur l'objectif de la Marche, l'activiste gay Predrag Azdejković a déclaré : « Un problème général de l'activisme LGBT serbe est qu'il aime mettre le chiffre 1 à côté de beaucoup de choses. Pour cette raison, ne serait-ce que l'année dernière, nous avons eu trois premières conférences régionales LGBT ». Razotkvirivanje, 15 avril 2015, en ligne: https:// groups.google.com/g/razotkvirivanje (consulté en juin 2020).

45. Communication personnelle, Belgrade, mai 2015.

46. KURTOVIĆ Larisa, «The Paradoxes of Wartime 'Freedom': Alternative Culture During The Siege of Sarajevo ", dans Bojan Bilić, Vesna Janković (dir.), Resisting The Evil: Post-Yugoslav Anti-War Contention, Baden-Baden, Nomos, 2012, p. 197-224 (220).

47. JANSEN Stef, Antinacionalizam: Etnografija otpora u Beogradu i Zagrebu [Anti-nationalisme : une ethnographie de l'opposition à Belgrade et à Zagreb], Belgrade, xx vek, 2005, p. 267.

48. Les «Femmes en noir» ont fait des efforts particuliers pour problématiser les distinctions entre urbain et rural dans l'espace politique serbe. Un nombre grandissant de projets féministes sont réalisés en dehors de la capitale. 
49. Entreprise américaine.

50. SAVIĆ Jelena, «Ženski marš iliti rijaliti šou solidarnosti » [Marche des femmes ou reality show de la solidarité], 2017, en ligne: http://proletter.me/portfolio/zenski-mars-iliti-rijaliti-sousolidarnosti/ (consulté en septembre 2020).

51. BILIĆ, STUBBS, « Unsettling 'the Urban' », art. cité.

52. JELENA, Razotkvirivanje, mai 2015, en ligne: https://groups.google.com/g/razotkvirivanje (consulté en juin 2020).

53. Certains activistes participant au débat se sont prononcés en faveur d'une différenciation entre séparatisme et exclusion. Tandis que le séparatisme devrait être pratiqué par des groupes ayant peu de pouvoir (par ex. lesbiennes, Noirs, etc.), pratiquer l'exclusion est le privilège de ceux qui ont davantage de pouvoir politique (par ex. les hommes). Cependant, cette discussion aboutit à une impasse si nous ne prenons pas en considération la proposition d'Yuval-Davis de compléter l'approche inter-catégorielle de l'oppression (lesbiennes, gays, Noirs, personnes en fauteuil roulant, etc.) par une approche intra-catégorielle (toutes les lesbiennes ne sont pas opprimées de la même façon, tous les hommes hétérosexuels ne sont pas privilégiés, etc.). Voir : YUVAL-DAVIS Nira, The Politics of Belonging: Intersectional Contestations, Londres, Sage, 2011.

54. MALjKović Dušan, Razotkvirivanje, mai 2015, en ligne: https://groups.google.com/g/ razotkvirivanje (consulté en juin 2020).

55. MLAĐENOVIĆ Lepa, Razotkvirivanje, mai 2015, en ligne: https://groups.google.com/g/ razotkvirivanje (consulté en juin 2020).

56. FRASER, « Rethinking the Public Sphere », art. cité.

57. GAMSON Joshua, « Must Identity Movements Self-Destruct? A Queer Dilemma », Social Problems, vol. 42, $\mathrm{n}^{\circ} 3,1995$, p. 390-407.

58. JANDRIĆ Ivana, "Institucionalni patrijarhat kao zakonitost kapitalizma " [Le patriarcat institutionnalisé en tant qu'ordre du capitalisme], 2018, en ligne : http://slobodnifilozofski.com/ 2018/12/institucionalni-patrijarhat-zakonitost.html (consulté en septembre 2020).

59. MIZIELIŃSKA Joanna, KULPA Robert, « 'Contemporary peripheries': Queer Studies, Circulation of Knowledge and East/West Divide », dans Robert Kulpa, Joanna Mizielińska (dir.), De-Centring Western Sexualities: Central and East European perspectives, Londres, Ashgate, 2011, p. 11-26 (15).

60. BILIĆ Bojan (dir.), LGBT Activism and Europeanisation in the Post-Yugoslav Space: On the Rainbow Way to Europe, Londres, Palgrave Macmillan, 2019.

\section{RÉSUMÉS}

La Marche des lesbiennes de 2015 à Belgrade a constitué le point culminant du mouvement séparatiste lesbien, fragile mais résistant, qui s'est développé dans l'espace (post-)yougoslave au cours des dernières décennies. Dans cet article, je m'appuie sur diverses sources empiriques pour analyser ce rassemblement en le plaçant dans le double contexte de l'histoire de l'organisation des féministes/lesbiennes yougoslaves et de celle, plus large, anglo-américaine, des mobilisations des militantes lesbiennes (et des GBT). J'examine les controverses que la Marche a provoquées parmi les militants pour aborder des questions plus vastes, telles que la manière dont l'oppression fondée sur le genre a été formulée et combattue après la fin du socialisme yougoslave. 
The 2015 Belgrade Lesbian March represented the culmination point of the fragile but resilient lesbian separatist movement that has been developing in the (post-)Yugoslav space over the last decades. In this paper, I draw upon a variety of empirical sources to reconstruct that march and contextualise it both in the history of Yugoslav feminist/lesbian organising as well as in the broader Anglo-American history of lesbian (and GBT) activist mobilisations. I use the controversies that the march provoked within the activist "community" to engage with more general concerns about issues of how gender-based oppression has been articulated and struggled against in the wake of Yugoslav socialism.

\section{INDEX}

Index géographique : Serbie, Yougoslavie

Keywords : Lesbian March, lesbian separatism, Serbia, Yugoslavia, post-socialism

Mots-clés : marche lesbienne, séparatisme lesbien, Serbie, Yougoslavie, postsocialisme

\section{AUTEURS}

\section{BOJAN BILIĆ}

Instituto de Ciências Sociais, Universidade de Lisboa

bojanello[at]gmail.com 